\title{
De la coopérative au groupe coopératif agro-alimentaire $1^{\text {re }}$ partie - De la valeur pour l'usage à la valeur pour
}

l'actionnaire?

\section{From the Cooperative to the Cooperative Group in the Food-} Processing Industry

\author{
Part One-A Shift from Value for Users to Value for \\ Shareholders?
}

\section{Michel Forestier et René Mauget}

Numéro 278, octobre 2000

Colloque Addes

ADDES Conference

URI : https://id.erudit.org/iderudit/1023849ar

DOI : https://doi.org/10.7202/1023849ar

Aller au sommaire du numéro

Éditeur(s)

Institut de l'économie sociale (IES)

ISSN

1626-1682 (imprimé)

2261-2599 (numérique)

Découvrir la revue

Citer cet article

Forestier, M. \& Mauget, R. (2000). De la coopérative au groupe coopératif agro-alimentaire : $1^{\text {re }}$ partie - De la valeur pour l'usage à la valeur pour l'actionnaire ? Revue internationale de l'économie sociale, (278), 16-30. https://doi.org/10.7202/1023849ar
Résumé de l'article

Cet article constitue la première partie d'une contribution, présentée au colloque Addes du 7 mars 2000, qui s'intitulait : « De la coopérative au groupe coopératif agro-alimentaire, évolution ou dénaturation ? " Les auteurs traitent notamment ici du glissement, dans les groupes coopératifs complexes, de la notion de valeur de la coopérative pour l'usager à la notion de valeur du groupe pour l'« actionnaire ». Y a-t-il dénaturation de l'entité coopérative ? Selon les critères retenus, la réponse à cette question pourra varier. La Recma publiera dans son prochain numéro la seconde partie de cette contribution, qui sera davantage centrée sur l'évolution de la gouvernance de ces groupes complexes. 


\section{DE LA COOPÉRATIVE AU GROUPE COOPERATIF AGRO-ALIMENTAIRE}

${ }^{\text {re }}$ partie: De la valeur pour l'usager à la valeur pour l'actionnaire?

$\left({ }^{*}\right)$ Michel Forestier est directeur de I'Aract Champagne-Ardenne. $\left({ }^{* *}\right)$ René Mauget est professeur et directeur à I'Essec-Imia. par Michel Forestier ${ }^{(*)}$ et René Mauget ${ }^{(* *)}$

Cet article constitue la première partie d'une contribution, présentée au colloque Addes du 7 mars 2000, qui sintitulait: "De la coopérative au groupe coopératif agro-alimentaire, évolution ou dénaturation?" Les auteurs traitent notamment ici du glissement, dans les groupes coopératifs complexes, de la notion de valeur de la coopérative pour l'usager à la notion de valeur du groupe pour l'" actionnaire ». Y a-t-il dénaturation de l'entité coopérative? Selon les critères retenus, la réponse à cette question pourra varier. La Recma publiera dans son prochain numéro la seconde partie de cette contribution, qui sera davantage centrée sur l'évolution de la gouvernance de ces groupes complexes.

es coopératives agricoles existent de moins en moins à l'état pur. Elles constituent des groupes, notamment lorsqu'elles transforment leurs produits, qui autour d'une coopérative articulent des filiales de statuts non coopératifs. Les filiales sont en outre souvent détenues en commun avec d'autres coopératives, voire des sociétés capitalistes industrielles ou bancaires. Se trouvent ainsi combinées dans les faits des logiques différentes, coopératives et capitalistes. Pour l'une, le facteur risqué est le produit; pour l'autre, il s'agit du capital.

Quelle est l'ampleur de ce mouvement de filialisation? Quels sont les critères d'efficacité retenus dans ces groupes? Qu'en est-il lorsque l'on fait un mariage entre ces deux types de société? Quelles évolutions en matière de paiement des agriculteurs, quelles conséquences par rapport aux finalités, aux valeurs et aux principes coopératifs? Autant de questions importantes pour l'avenir de ce type d'organisation.

Notre analyse portera sur les groupes coopératifs français. Elle pourrait globalement être étendue aux groupes européens, mais les précisions données dans la présente contribution s'appliquent à l'environnement juridique français, qui est sensiblement différent des pays du nord de l'Europe, et les évolutions structurelles sont plus avancées en France qu'en Europe du Sud. 


\section{Observation des mouvements quantitatifs et structurels}

\section{Exemple d'évolution quantitative sur une dizaine d'années: la Cana (44)}

\begin{tabular}{|c|c|c|c|c|c|c|}
\hline \multirow[t]{2}{*}{ Indicateurs } & \multicolumn{3}{|c|}{1985} & \multicolumn{3}{|c|}{1997} \\
\hline & Coopérative & $\begin{array}{l}\text { Coopérative } \\
\text { en } \% \text { groupe }\end{array}$ & Groupe & Coopérative & $\begin{array}{l}\text { Coopérative } \\
\text { en } \% \text { groupe }\end{array}$ & Groupe \\
\hline CA en millions de francs & 3161 & $73 \%$ & 4392 & 3266 & $34 \%$ & 9635 \\
\hline VA en $\%$ du chiffre d'affaires & $10,20 \%$ & & $9,80 \%$ & $6,7 \%$ & & $12 \%$ \\
\hline $\begin{array}{l}\text { Immo. corporelles } \\
\text { (en millions de francs) }\end{array}$ & 282 & $75 \%$ & 311 & 130 & $21 \%$ & 617 \\
\hline $\begin{array}{l}\text { Nombre de salariés } \\
\text { (en unités) }\end{array}$ & 1512 & $78 \%$ & 1940 & 1128 & $25 \%$ & 4516 \\
\hline Contrôle du groupe & $100 \%{ }^{(1)}$ & & & $91^{(2)}$ & & \\
\hline
\end{tabular}

A la Cana, la filialisation massive a été effectuée en 1989, après l'émission de titres participatifs et l'entrée en Bourse de 1988. Aujourd'hui, environ un tiers du chiffre d'affaires est effectué dans la coopérative, contre les trois quarts en 1985 . Il n'y a plus que $25 \%$ des salariés dans la coopérative, alors qu'en 1985 ils étaient plus des trois quarts. Le glissement sur les immobilisations corporelles est encore plus important. Quant à la valeur ajoutée, elle a bien sûr glissé avec les autres indicateurs, mais il est intéressant de souligner qu'en 1985 les activités filialisées étaient à plus faible valeur ajoutée que la moyenne du groupe, alors qu'aujourd'hui les activités à forte valeur ajoutée sont filialisées. Il ne reste dans la coopérative que les activités d'amont (achat et vente avec agriculteurs, notamment).

Le phénomène que nous présentons sur la Cana n'est pas unique, il est représentatif de ce que nous retrouverons dans de nombreux groupes coopératifs agro-alimentaires. 


\section{Exemples d'évolution structurelle}

Ces évolutions structurelles sont anciennes. On les voit apparaître dès les années 60-70 à l'ULN ou à la Cecab, par exemple. Cependant, ces mutations se sont généralisées depuis les années 80 .

\section{Le schéma générique}

Schéma type de groupe coopératif

Partenaires financiers

$\downarrow$



Maison mère coopérative

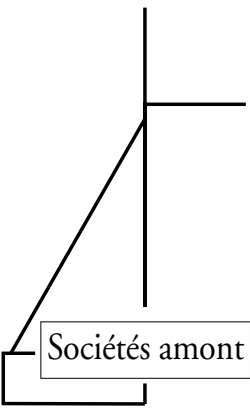

Holding activité aval

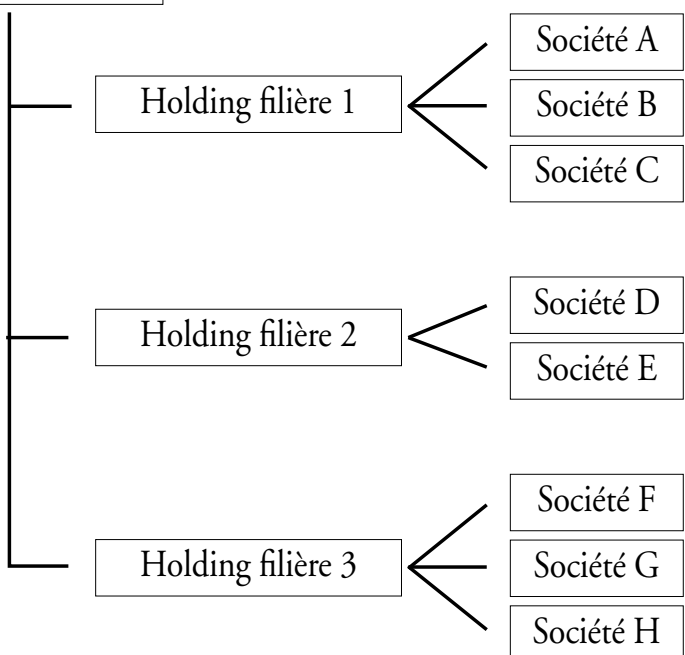


Le schéma ci-après rend compte de la structure du groupe Cana (1998).

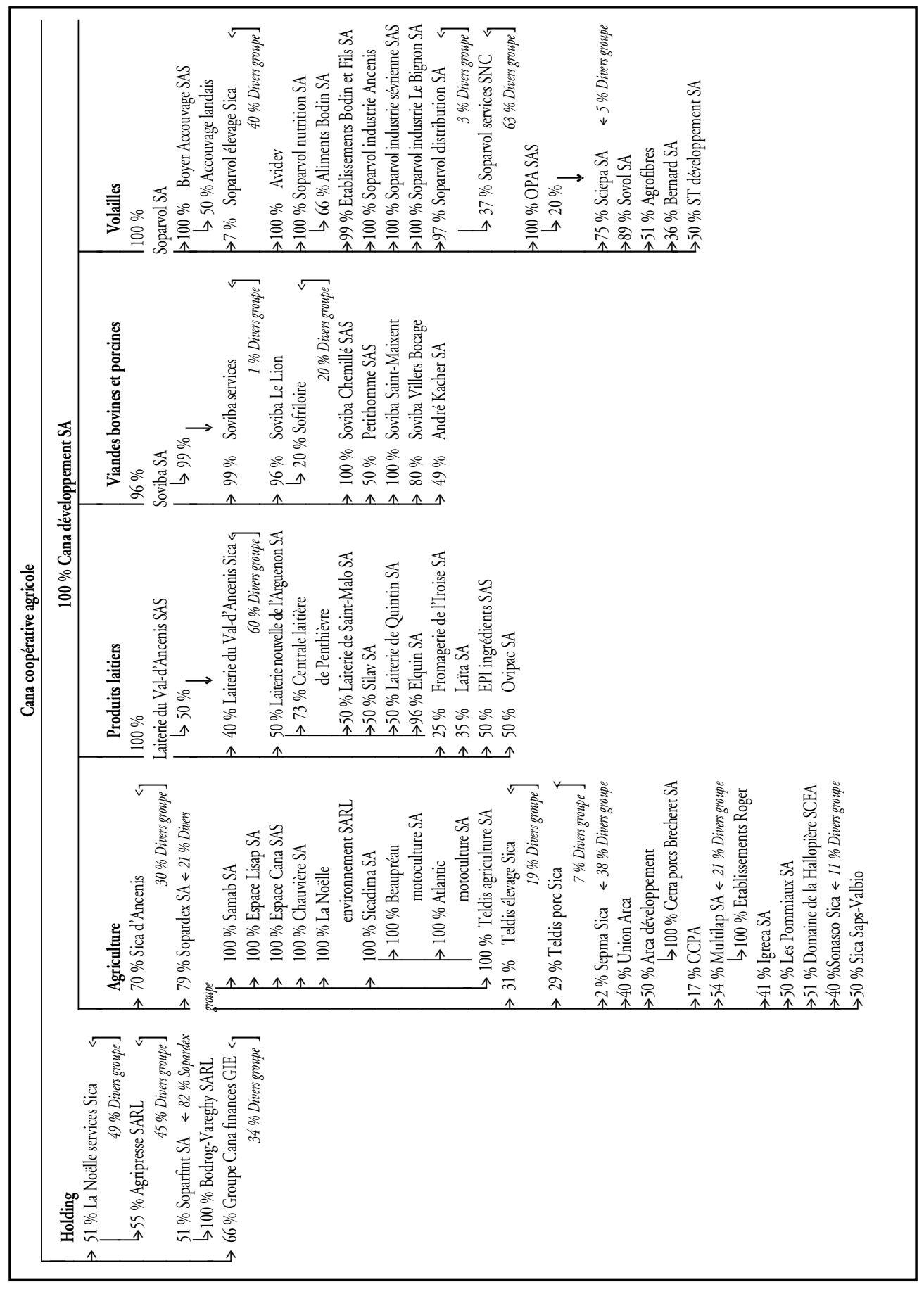


(1) Voir deuxième partie de cette contribution, à paraître dans le prochain numéro de la $\operatorname{Recma}\left(\mathrm{n}^{\circ} 279\right)$.

(2) Voir les travaux de Chandler.
Ces évolutions structurelles engendrent de multiples conséquences qui sont plus ou moins importantes selon les coopératives.

Dans le cas de la Cana, le mode d'organisation et de fonctionnement permet de conserver un bon contrôle par la société mère. Dans quelques autres cas, ce contrôle est nettement moins rigoureux.

Dans le groupe Transagra, aujourd'hui disparu, la mère principale était la coopérative Agri Cher. Le contrôle du groupe dans ce cas de figure, grâce à un holding sous forme de commandite par action, avait été repris par plusieurs personnes, dont une société d'investissements cotée en Bourse. Les conséquences seront donc différentes en fonction des modes d'organisation, de la volonté des dirigeants et des partenaires ${ }^{(1)}$.

Remarque: Les groupes filialisés sont le plus souvent des groupes agro-alimentaires, c'est-à-dire des coopératives qui ont choisi des stratégies d'intégration verticale. La concentration sur le métier de base de collecte nécessite en général moins de capitaux et de partenariat avec le secteur non coopératif. Dans ce cas, le groupe complexe n'est pas indispensable; une coopérative intégrée ou une union de coopérative peut suffire le plus souvent. Ce n'est pas l'objet de cette contribution d'analyser la pertinence d'une stratégie d'intégration verticale par rapport à une stratégie de concentration sur la collecte (type Opep). Mais le choix de stratégie a incontestablement des influences sur le choix de structure ${ }^{(2)}$.

\section{Pourquoi ces évolutions?}

Avant d'analyser les conséquences, il est intéressant de revenir sur le pourquoi de ces évolutions. Nous avons identifié au moins cinq types de raisons.

\section{Des raisons stratégiques consécutives à une globalisation des marchés}

Cette globalisation a presque toujours eu comme conséquence une concentration, notamment commerciale, mais aussi une nécessité de réduire les coûts et (ou) de différencier les produits.

\section{Des raisons juridiques}

- Contraintes statutaires limitant la capacité commerciale (règle de l'exclusivisme et de territorialité, notamment).

- Contraintes pour faire des alliances entre le secteur privé et la société mère coopérative.

\section{Des raisons financières}

- Difficulté pour mobiliser les capitaux propres avec la société mère, ce qui entraîne le développement des holdings.

Des raisons fiscales parfois

- Intérêt de la structure holding pour consolider les résultats des filiales. 
Des raisons de management, de prise de décision et de gestion

- Simplification, rapidité, meilleure vision des rentabilités par métier, recentrage facilité, etc.

Il convient cependant de noter que la filialisation n'est pas la réponse unique à ces problèmes, ni une panacée.

\section{En résumé}

Le but de cette évolution était et est toujours d'assurer la survie et (ou) d'accroître l'efficacité du groupe coopératif face à l'accroissement de la dynamique concurrentielle. Aujourd'hui, après une dizaine d'années de filialisation, il est intéressant de s'interroger sur les conséquences de ces mouvements.

\section{Définition des concepts utilisés pour l'analyse}

(3) Q: quantité. PP: prix des pro duits. Qch: quantité des charges. Pch: prix des charges (engrais, phyto, services...).

\section{Apport de valeur par la coopérative pour l'usager}

Nous prendrons l'exemple d'une coopérative d'écoulement (collecte et transformation).

\section{L'avantage coopératif à court terme}

- Avantage à court terme sur le prix.

Prix coopératif - Prix de marché = Avantage à court terme sur le prix.

- Avantage à court terme sur le revenu.

La coopérative peut intervenir sur le revenu de trois manières: avantage prix (voir ci-dessus); avantages quantité (droit à produire, effet de productivité sur l'exploitation agricole); avantages par la maîtrise des charges sur les exploitations agricoles.

Revenu $=\mathrm{Q} \times \mathrm{PP}-\mathrm{Qch} \times \mathrm{Pch}{ }^{(3)}$.

- Sécurité de paiement.

Il est en effet très rare qu'en cas de faillite ou de difficultés l'engagement de paiement des produits par la coopérative ne soit pas respecté. La coopérative est le plus souvent reprise par une autre coopérative qui respecte les engagements de paiement. Il n'en est pas toujours de même dans le cas d'une faillite d'entreprise non coopérative.

\section{Remarque sur la fixation des prix à court terme}

- Le prix coopératif (PC).

$\mathrm{PC}=$ Acompte + Compléments de prix + Ristourne.

Cette grandeur est à comparer au prix de marché pour observer s'il y a un avantage coopératif à court terme. Pour que cette comparaison soit pertinente, il faudrait en même temps que la coopérative rémunère le capital engagé, au prix de marché des capitaux non risqués (ou opérer un retraitement comptable).

- Le prix de marché des produits.

Ce dernier n'est pas facile à déterminer comme élément de comparaison. 
(4) VC: valeur créée (economic value added). $\mathrm{RCl}$ : rentabilité des capitaux investis en pourcentage (résultat opérationnel moins I'impôt). CCE : coût des capitaux engagés en pourcentage. $\mathrm{Cl}$ : capitaux investis (valeurs immobilisées nettes plus $B F R$ ).
En effet, il dépend souvent du prix d'acompte de la coopérative lorsque celle-ci est leader sur son marché (par exemple en céréales), car dans ce cas les entreprises non coopératives font le même prix.

Dans plusieurs productions (lait ou légumes transformés, par exemple), il existe un prix régional ou national qui est la résultante de négociations interprofessionnelles comprenant des syndicats paysans, les coopératives, les industriels privés. Il s'agit plutôt d'un " prix politique » qui peut se négocier une fois par an.

Ainsi, en théorie, le calcul de l'avantage coopératif pour l'usager est simple, mais dans la pratique cet avantage est difficile à évaluer de façon précise.

\section{L'avantage coopératif à long terme}

L'avantage coopératif se manifeste à long terme par un moindre risque de délocalisation de la production, donc du revenu de l'agriculteur. En effet, les critères de décisions sont différents de ceux de l'entreprise non coopérative (ENC).

Pour une ENC multirégionale ou multinationale, le raisonnement rationnel est le suivant: peut-on trouver une meilleure valorisation de capitaux sur le long terme en délocalisant l'activité?

Dans le cas de l'entreprise coopérative, la délocalisation, peu fréquente, ne s'effectue qu'après avoir épuisé les solutions économiques dans la région d'origine (l'avantage pour l'usager en termes de revenu l'emporte sur la notion de rentabilité des capitaux découlant d'une éventuelle délocalisation).

Néanmoins, dans le cadre de raisonnements complexes, le groupe coopératif peut être amené à effectuer son expansion en dehors de son territoire, mais ce n'est que rarement une décision de premier choix.

\section{Apport de valeur par le groupe coopératif pour l'actionnaire}

Nous définirons d'abord ce concept dans le cadre de l'entreprise capitaliste, puis nous verrons si la même approche peut être retenue dans les groupes coopératifs complexes.

\section{La création de valeur dans les entreprises capitalistes}

Ce concept est devenu une obsession pour de nombreux investisseurs et dirigeants d'entreprise. C'est un peu la dictature du court terme, disent certains chefs d'entreprise, et cette approche ne serait pas très bien adaptée à certains investissements nécessaires sur le long terme et pourtant créateurs de valeur à long terme.

- Définition du concept.

La valeur créée par une entreprise capitaliste correspond à la différence entre la rentabilité des capitaux investis et le coût des capitaux engagés.

$\mathrm{VC}=(\% \mathrm{RCI}-\% \mathrm{CCE}) \times \mathrm{CI}^{(4)}$.

\section{La création de valeur pour l'actionnaire dans le groupe coopératif}

La façon d'appréhender ce concept dans une coopérative est un peu plus complexe. 
- Cas d'une filiale qui transforme des produits ne provenant pas des associés (filiales délocalisées, par exemple).

Dans ce cas de figure, le raisonnement que nous avons évoqué ci-dessus peut être pertinent à condition que cet investissement n'ait pas d'effet négatif sur la valeur dans le groupe coopératif en France pour l'usager.

- Cas d'une filiale qui transforme des produits des sociétaires.

Dans ce cas, il convient d'étudier la façon dont a été déterminé le prix de marché pour le produit de base. Si celui-ci a été convenablement évalué par rapport à l'environnement, le raisonnement précédent peut encore être pertinent.

\section{Remarque}

Dans ces deux cas de figure, il y a ensuite plusieurs façons d'appréhender le résultat.

- Au niveau de la société mère coopérative.

L'investissement dans la filiale a le plus souvent été effectué par la société mère coopérative. Cette dernière peut se contenter du raisonnement précédent.

Cependant, ce raisonnement reste simpliste, car une optimisation des droits à produire pour les agriculteurs peut entraîner une baisse de la rentabilité au sein de la filiale, donc une diminution des dividendes avec avoir fiscal, mais une augmentation globale de la recette pour les agriculteurs: Q x P. Pour déterminer l'avantage coopératif pour l'agriculteur, il conviendra donc de faire une analyse consolidée de l'avantage (coopérative plus agriculteurs) comparée à l'ancienne situation, avant augmentation des droits à produire. - Au niveau des associés coopérateurs qui sont les propriétaires de la société mère.

A ce stade l'analyse est plus complexe.

Sur le long terme, ce que veut l'intéressé, c'est la pérennité et l'augmentation de ses droits à produire, de son activité d'agriculteur.

Sur le court terme, l'agriculteur dans la coopérative classique était intéressé par un sur-prix ou un sur-revenu en provenance de sa coopérative par rapport à l'agriculteur qui travaillerait avec une entreprise non coopérative (dans la réalité, ce n'est pas toujours le cas).

L'organisation en groupe complexe filialisé doit donc permettre de verser à l'associé coopérateur un montant au moins équivalent au montant perçu dans le cas de la coopérative classique. Cependant, celui-ci sera versé sous une forme différente, à savoir : prix de marché (PM) plus dividendes avec avoir fiscal (DAF), versés au prorata du capital détenu par le sociétaire dans sa coopérative (en France, ceci est possible depuis la loi du 3 janvier 1991). Ainsi, la valeur du groupe coopératif pour le coopérateur actionnaire à court terme se limite au pourcentage de dividendes versés par rapport au capital détenu par lui, ce montant s'ajoutant au prix de marché qu'il aura perçu antérieurement. A cela peut s'ajouter la rémunération du capital social du sociétaire, qui, nous l'avons dit, pourrait se situer au niveau des obligations sans risque comme dans la coopérative classique, pour que cette variable 
soit neutralisée dans la comparaison. Nous rappelons que l'associé coopérateur n'a pas, en théorie, de droits sur les réserves.

- Cas où l'agriculteur se situe à deux niveaux de pouvoir et de financement. L'agriculteur peut recevoir au niveau de la société mère, comme précédemment:

$(\mathrm{PM}+\mathrm{DAF})+\mathrm{i} \%$ sur parts sociales.

Au niveau de la filiale, il peut investir directement. Aujourd'hui, plusieurs coopératives ont ainsi ouvert le financement des filiales aux agriculteurs (par exemple, Epis-Centre avec Maltor). Dans ce cas de figure, en plus des recettes précédentes, il faut ajouter les dividendes perçus à ce niveau et les perspectives de plus-values.

De ce qui précède on voit que l'analyse de l'avantage coopératif pour l'usager et (ou) pour l'actionnaire dans un groupe coopératif est très complexe.

\section{Synthèse des conséquences de ces mutations sur le plan économique et par rapport à la doctrine coopérative}

(5) En droit, deux fois le capital social.

(6) Dans la pratique, la loi de 1972 permet en France de revaloriser le capital social avec les réserves disponibles, dans la limite de l'évolution de la rente viagère.

\section{Les conséquences en matière de rémunération des agriculteurs et de critères d'efficacité}

\section{Dans la coopérative agricole classique}

Dans la coopérative agricole classique, le facteur risqué est le produit, alors que dans une entreprise capitaliste, il s'agit du capital. Le capital social dans la coopérative est plus proche d'un capital obligation ou d'un prêt des membres que d'un capital action. Il subit néanmoins le risque de faillite ${ }^{(5)}$. Les implications stratégiques et financières de cette forme d'organisation sont donc déterminantes.

Le capital bénéficie d'une rémunération limitée pouvant aller de zéro jusquau taux moyen des obligations (en France). Les sociétaires n'ont pas de droit sur les réserves ${ }^{(6)}$. En revanche, les surplus dégagés par l'exploitation peuvent être distribués aux adhérents sous forme de ristournes au prorata des transactions économiques avec la coopérative. C'est le principe " acapitaliste ».

Les différentes variables du compte de résultat, valeur ajoutée, excédent brut d'exploitation (EBE), capacité d'autofinancement (CAF), résultat net, n'ont ainsi pas la même signification que dans une entreprise capitaliste. Les produits apportés par l'adhérent dans la coopérative constituent une charge dans le compte de résultat de la coopérative, mais il s'agit en réalité d'une charge à maximiser (acompte plus complément de prix), car elle constitue, avec la ristourne, la variable à maximiser sur le long terme dans la coopérative (facteur risqué).

Ainsi, nous voyons que la présentation comptable classique n'est pas pertinente pour juger de l'efficacité de la coopérative d'écoulement. Des retraitements comptables sont souvent nécessaires pour juger de l'efficacité de 
(7) Voir note (6), en page précédente.

(8) Selon la théorie de l'agence. la coopérative pour l'adhérent. En effet, la valeur de la coopérative pour l'adhérent se manifeste d'abord par l'équation:

(Acompte + Complément de prix + Ristournes) x Quantités produites. A cela on peut ajouter la rémunération du capital social.

L'approche patrimoniale de la coopérative pour l'adhérent, par le bilan, est également plus complexe que dans l'entreprise capitaliste. En effet, l'adhérent n'a pas de droits sur les réserves, il n'a pas droit aux plus-values. La valeur de son capital reste à la valeur nominale, comme dans le cas d'un prêt ou d'un compte associé (7); il n'a pas droit non plus à des dividendes. La situation nette de la coopérative ne représente pas le patrimoine des propriétaires de la coopérative comme dans l'entreprise capitaliste. Le ratio fondamental utilisé dans l'entreprise capitaliste (résultat net-fonds propres) n'est pas pertinent pour déterminer la création de valeur par la coopérative pour l'adhérent, car le numérateur est le fruit de décisions politiques et le dénominateur n'appartient pas en totalité au coopérateur.

Nous verrons que, dans un groupe coopératif avec partenariat capitaliste, ce ratio peut retrouver une légitimité. Cependant, les grands équilibres économiques et financiers de la coopérative gardent un sens pour la rémunération des produits des adhérents sur le moyen et sur le long terme (pérennité de la coopérative et capacité de développement).

\section{Les critères d'efficacité retenus dans un groupe complexe}

Les implications en matière de management stratégique et financier sont importantes pour la coopérative et pour l'agriculteur. En effet, dans un groupe complexe, surtout lorsqu'il y a des partenaires non coopératifs, qu'ils soient financiers (banque) ou industriels, nous avons un mariage entre deux entités qui souhaitent optimiser un facteur risqué différent: les agriculteurs, le produit; le partenaire capitaliste, le capital.

Il conviendra donc d'effectuer un arbitrage et, dans le cas où la coopérative demande l'alliance, elle sera amenée à modifier son comportement, même si elle est en position majoritaire ${ }^{(8)}$.

Les études que nous avons conduites auprès de groupes coopératifs complexes nous montrent une évolution significative dans la rémunération du produit et notamment dans la sphère industrielle et commerciale filialisée. Pour résumer, la rémunération du produit dans cette sphère évolue de la façon suivante.

- Rappel coopérative classique.

Acompte + Complément de prix + Ristournes.

- Sphère aval dans les groupes complexes (le résultat est externalisé).

Prix de marché + Dividendes, avec possibilité, depuis la loi du 3 janvier 1991 en France, de faire une remontée des dividendes avec avoir fiscal jusqu'aux sociétaires.

Il reste que la société mère peut encore effectuer des arbitrages, au sein de la coopérative mère, entre intérêt sur le capital social, dividendes avec avoir fiscal et ristournes, ce que nous retrouvons aujourd'hui dans la pratique de ces groupes. 
En ce qui concerne l'arbitrage entre autofinancement et dividende dans les filiales en partenariat avec le privé, là aussi la coopérative est souvent amenée à modifier son comportement. Pour éviter les conflits chaque année, les partenaires définissent alors une règle de décision, ce qui limite le conflit, mais n'est pas toujours d'une grande pertinence économique quant à l'arbitrage annuel entre autofinancement et distribution.

\section{Intérêts de ces évolutions}

Nous avons vu que la motivation à créer ces groupes était surtout stratégique et financière pour s'adapter à un nouvel environnement concurrentiel, la coopérative classique n'ayant pas la souplesse nécessaire. Il convient donc maintenant d'analyser l'intérêt de ces évolutions pour l'agriculteur sociétaire et les conséquences au regard des finalités, des valeurs et de la doctrine coopérative.

\section{Quelle création de valeur pour l'usager et/ou l'actionnaire?}

La réponse à cette question sera difficile, plus difficile à construire que ne l'est aujourd'hui l'amélioration de la valeur pour l'actionnaire dans l'entreprise non coopérative.

Dans une société cotée en Bourse, on voit assez rapidement l'effet d'une stratégie ou d'une politique ayant pour but l'amélioration de la valeur pour l'actionnaire. L'actualité récente nous montre que les recentrages (Danone, par exemple), les concentrations (Carrefour, Promodès), les licenciements (Michelin) et les rachats d'actions par l'entreprise ont souvent eu un effet positif dans l'augmentation à court terme de la valeur pour l'actionnaire.

Dans le cas des évolutions stratégiques et structurelles des groupes coopératifs, l'effet sur l'usager et l'apporteur de capital qu'est l'agriculteur n'est pas aussi simple et facile à quantifier.

La valeur pour l'usager est parfois difficile à évaluer (voir ci-dessus).

La valeur pour l' " actionnaire " prend aujourd'hui une signification depuis que l'on peut reverser des dividendes avec avoir fiscal et éventuellement des plus-values au prorata du capital investi jusqu'aux associés. Néanmoins, ce résultat positif pour l' " actionnaire " serait à comparer à un éventuel manque à gagner sous forme de ristournes pour l'usager.

Ainsi, on pourrait concevoir une stratégie de délocalisation ou d'expansion hors région (c'est déjà une réalité dans certaines productions) qui permettrait de bénéficier de faibles coûts d'intrants et de salaires pour augmenter les profits, lesquels seraient ensuite distribués aux adhérents du groupe coopératif, au prorata du capital social. La valeur pour l'actionnaire prendrait ainsi une place plus importante, mais celle-ci serait à comparer à une éventuelle diminution des « droits à produire " pour les adhérents dans leur région ou pays d'origine.

Dans le cadre de la globalisation des marchés et de l'internationalisation des concurrents, cette réflexion devient pertinente pour l'agriculteur " actionnaire " et usager. Faut-il pour une coopérative aller faire du foie gras en Bulgarie ou en Chine? Faut-il aller produire du poulet au Brésil pour les 
marchés du Moyen-Orient ou d'ailleurs? C'est possible, ça peut rapporter des dividendes, mais il convient d'en analyser les conséquences pour l'usager et d'en faire le bilan global.

Deux exemples nous permettront d'illustrer la complexité du mode de raisonnement du groupe coopératif: celui de Malteurop et celui d'Unicopa. - Le groupe Malteurop.

Le groupe Malteurop, filiale de groupes coopératifs, s'est installé en Chine. Premier niveau de raisonnement: Pourquoi investir de l'argent en Chine pour ouvrir une usine là-bas au lieu d'exporter du malt avec des céréales françaises?

Deuxième niveau de raisonnement: Si le groupe coopératif n'investit pas à l'étranger, il risque de ne pas conserver ses parts de marché à l'exportation (coût du malt), car des concurrents investiront en Chine et produiront à moindre coût.

Troisième niveau de raisonnement: Si Malteurop ne va pas en Chine, non seulement il perdra le marché chinois, mais il risque de perdre des marchés ailleurs, car les brasseurs internationaux peuvent se lier avec des malteurs concurrents et abandonner progressivement Malteurop, qui sera marginalisé car non implanté à l'international. Donc, il faut investir en Chine.

Quatrième niveau de raisonnement: Il s'agit pour l'agriculteur champenois d'un moindre mal et il peut compenser son manque à gagner en termes d'usager par un plus, nous le verrons, sous forme de dividendes avec avoir fiscal si l'opération en Chine est rentable. La perte potentielle pour l'usager sera compensée en partie par un avantage pour l'actionnaire.

Ainsi, nous voyons que l'internationalisation est perçue par l'entreprise non coopérative (ENC) comme une solution de premier choix, alors que dans la coopérative elle ne vient qu'après une série de réflexions complexes et en privilégiant la notion de valeur de la coopérative pour l'usager. Souvent l'expansion hors région est repoussée lorsque celle-ci est à prendre par un conseil d'administration composé d'agriculteurs.

- Unicopa.

Cet exemple permettra d'illustrer le refus d'internationalisation.

Exemple: cas des poulets pour le marché du Moyen-Orient.

Exportateurs: Doux (groupe non coopératif) et Unicopa (groupe coopératif).

Choc: baisse des restitutions à l'exportation dans le cadre du Gatt, période 1994-2000.

Stratégies des deux protagonistes:

- L'entreprise non coopérative Doux va rechercher à se délocaliser là où les coûts de production sont inférieurs. Par exemple, l'indice du coût de production est de 100 au Brésil, alors qu'il est de 185 en France sur l'ensemble de la filière. Doux ira donc s'implanter au Brésil, ce qu'il a déjà réalisé avec Frangosul. Cette internationalisation lui permet de récupérer le marché du Moyen-Orient et peut-être d'en conquérir de nouveaux.

- Que va faire Unicopa? Le groupe coopératif peut-il raisonner de la même manière? Les effets sur les filières volailles françaises en direction 
du Moyen-Orient sont les mêmes à terme: fermeture d'usines en Bretagne et diminution de ce type de production chez les agriculteurs bretons. En revanche, la décision d'internationalisation ne sera sans doute pas prise dans ce dernier cas, car trop éloignée des finalités de maintien du revenu des adhérents d'Unicopa, et l'on envisage difficilement de demander des fonds aux agriculteurs de ce groupe pour investir au Brésil. La solution recherchée ira dans la direction d'un changement de production pour le marché européen, même si celui-ci est aujourd'hui très " engorgé ». On peut même aller vers une solution plus radicale (arrêt de l'activité).

\section{Conséquences par rapport aux finalités, aux valeurs et à la doctrine}

\section{Au regard des finalités}

Il convient de définir les critères: le prix par unité produite; le revenu à partir de la production agricole; les droits à produire (nombre d'unités produites par marché); le revenu financier quelle qu'en soit l'origine (revenu des produits agricoles et revenu financier des filiales, quelle que soit la localisation). La réponse sur l'atteinte des buts dépendra des critères retenus. Le dernier critère est encore peu utilisé, mais certains groupes communiquent sur ce point.

\section{Au regard des valeurs}

Hier, les valeurs le plus souvent avancées par les coopératives étaient: la solidarité; l'égalité (souvent prix unique); la transparence.

Aujourd'hui, les valeurs ont souvent évolué, les coopératives formulant fréquemment: l'équité (un service égale un coût égale un prix), qui a remplacé l'égalité, laquelle serait de moins en moins acceptée par les sociétaires; la transparence; la solidarité sélective (jeunes, agriculteurs en difficulté, etc.).

Le groupe bien contrôlé par les agriculteurs peut permettre de respecter ces valeurs. La présence de partenaires capitalistes (financiers ou industriels) pourrait néanmoins faire pression sur les dirigeants de la coopérative pour effectuer une sélectivité des adhérents en fonction de critères de rentabilité économique et rompre avec les valeurs d'égalité et même d'équité.

\section{Au regard de la doctrine (les principes coopératifs)}

Les principes coopératifs sont essentiellement: l'acapitalisme (principe de la ristourne, pas de dividendes, pas de droit aux plus-values, intérêts limités); la démocratie coopérative (le plus souvent, " un homme égale une voix "); le double exclusivisme (la coopérative ne peut vendre que les produits de ses adhérents, ceux-ci s'engageant de leur côté à livrer toute leur production); la porte ouverte (liberté d'entrée et de sortie, avec engagement réciproque).

Dans les groupes coopératifs avec alliance, lorsque la valeur ajoutée est réalisée dans les filiales de droit commun, les principes coopératifs sont bousculés. 
- L'acapitalisme est le principe le plus remis en question. En effet, le prix de marché est souvent la règle et le surplus peut être redistribué sous forme de dividendes au prorata du capital. Le mode de paiement est le même que si l'agriculteur était actionnaire d'une société de droit commun.

- La démocratie coopérative " un homme égale une voix " peut être respectée dans la société mère, mais le pouvoir sur les filiales est en général au prorata du capital de chaque associé.

- L'exclusivisme dans les filiales n'est pas obligatoire et la filiale peut travailler avec qui elle veut. C'est un choix stratégique entre un système intégré exclusif ou un système pseudo-intégré dans lequel la filiale et la coopérative restent libres et font jouer la concurrence (ceci est fréquent dans la viande ou l'alimentation animale, notamment).

- La porte ouverte: ce principe peut être respecté, mais la pression du partenaire non coopératif peut conduire la coopérative à des décisions allant à l'encontre de ce principe, par exemple en rejetant des agriculteurs non performants, soit en termes de qualité, soit en termes de coût pour la filiale. Au regard de cette analyse on peut remarquer que si les finalités, les valeurs et les principes sont touchés par cette évolution, c'est le principe de base, l'acapitalisme, qui est le plus remis en question. Peut-on dire pour autant qu’il s'agit d'une dénaturation? La réponse à cette question dépendra évidemment des critères de jugement que l'on privilégiera. Faut-il subordonner les principes coopératifs aux décisions du niveau "supérieur ": les finalités et les valeurs? Les pays du nord de l'Europe ont souvent fait ce choix. Les pays du sud de l'Europe raisonnent parfois de façon différente. En France, aujourd'hui, la tendance est plutôt à hiérarchiser: 1) Finalités; 2) Valeurs; 3) Principes coopératifs.

Il convient de rappeler qu'en France, comme dans de nombreux pays, les coopératives sont plus des «filles de la nécessité » que des montages doctrinaires. Cette genèse explique sans doute qu'aujourd'hui le bouleversement des principes coopératifs ne traumatise ni la plupart des agriculteurs ni la majorité des dirigeants de coopérative, tant que le cap des finalités est globalement respecté. Le management de ces groupes complexes et importants en taille effraie souvent plus que l'abandon de l'acapitalisme. Cet aspect sera développé dans le prochain numéro. 


\section{Bibliographie sélective}

\section{Ouvrages}

Caby J. et Hirigoyen G., La création de valeur de l'entreprise, Economica, 1997.

Charraux G., Le gouvernement des entreprises théories et faits, Economica, 1977.

Deshayes G., Logique de la coopération et gestion des coopératives, Skippers 1988.

Mauget R., Quelles stratégies pour les coopératives agricoles, thèse, Paris-I, 1982.

Mevellec P., Belley M., Gestion financière dans les organisations coopératives, Ed. Gaëtan Morin, 1985.

Nilsson J., Dijk G. V., Strategies and Structures in the Agro-food Industries, Ed. Van Gorcum, 1997.

Pichette C., Analyse micro-économique et coopérative, Sherbrooke (Québec), Les Cahiers de la coopération, 1972.

Vienney C., Socio-économie des organisations coopératives, tome 1 et 2, Ciem, "Tiers secteur ", 1980.

\section{Articles}

Champagne $\mathbf{P}$., « Les administrateurs de coopératives sont-ils indispensables? », Recma, $\mathrm{n}^{\circ} 269$. Forestier M., « Du besoin d'administration des coopératives ", Recma, n 269.

Mauget R. et Declerck F., «L'analyse stratégique des groupes coopératifs européens ", Economie et Gestion agro-alimentaire, octobre 1993. Mauget R., Evolution des coopératives durant les trente dernières années, approche statistique et stratégique: l'entreprise coopérative expérience et recherche francophone, Ed. Pulim, 1998.

Mauget R., "La course à la taille », Euralis info, décembre 1999.

Mauget R., «Le partenariat coopératives-firmes privées ", Economie et Gestion agro-alimentaire, avril 1994.

Mauget René, «Les moyens de financement des groupes coopératifs agro-alimentaires et leurs conséquences ", Recma, n 242.

Nicolas Philippe, « La formation des groupes coopératifs agro-alimentaires et la situation des agriculteurs sociétaires ", Recma, n 34 . 